\title{
Congenital Adrenal Hyperplasia in Children: A Pilot Study of Steroid Hormones Expressed as Sex- and Age-Related Standard Deviation Scores
}

\author{
Caroline S. Clausen ${ }^{a}$ Marie L. Ljubicic ${ }^{a}$ Katharina M. Main ${ }^{a}$ \\ Anna-Maria Andersson ${ }^{a}$ Jørgen H. Petersen ${ }^{b}$ Hanne Frederiksen ${ }^{a}$ \\ Morten Duno $^{c}$ Trine H. Johannsen ${ }^{a}$ Anders Juul ${ }^{a}$ \\ a Department of Growth and Reproduction and International Center for Research and Research Training in \\ Endocrine Disruption of Male Reproduction and Child Health (EDMaRC), Rigshospitalet, University of Copenhagen, \\ Copenhagen, Denmark; ${ }^{b}$ Department of Biostatistics, University of Copenhagen, Copenhagen, Denmark; \\ 'Department of Clinical Genetics, Rigshospitalet, Copenhagen, Denmark
}

\section{Keywords}

Congenital adrenal hyperplasia $\cdot 21$-Hydroxylase deficiency . Adrenal metabolites - Standard deviation scores · Adrenal gland

\begin{abstract}
Introduction: Congenital adrenal hyperplasia (CAH) is an autosomal recessive disease predominantly caused by 21-hydroxylase deficiency. Clinical management in children includes glucocorticoid and often mineralocorticoid treatment alongside monitoring outcomes such as anthropometry, pubertal status, blood pressure, and biochemistry. Objective: The objective of this pilot study was to present the use of 17-hydroxyprogesterone (17-OHP) and androgen metabolites expressed as standard deviation (SD) scores rather than actual concentrations as a tool in the management of children with $\mathrm{CAH}$ as well as in research settings. Methods: The study was a retrospective, longitudinal study that took place in a single, tertiary center and included 38 children and adolescents aged 3-18 years with CAH due to 21-hydroxylase deficiency. Biochemical measurements of 17-OHP, androstenedione, dehydroepiandrosterone-sulphate (DHEAS), and testosterone using liquid chromatography-tandem mass spectrometry were expressed as SD
\end{abstract}

scores, and outcomes such as genotype, height, bone maturation, blood pressure, and treatment doses were extracted from patient files. Results: The majority (86\%) of CAH patients had 17-OHP measurements above +2 SD during standard hydrocortisone therapy, receiving an average daily hydrocortisone dose of $12.6 \mathrm{mg} / \mathrm{m}^{2}$. Androstenedione concentrations were mostly within $\pm 2 \mathrm{SD}$, whereas DHEAS values were below -2 SD in $47 \%$ of patients. Conclusions: Applying sex- and age-related SD scores to 17-OHP and androgen metabolite concentrations allows for monitoring of hydrocortisone treatment independent of age, sex, assay, and center. We propose that 17-OHP and androgen metabolites expressed as SD scores be implemented as a unifying tool that simplifies research and, in the future, also optimal management of treatment.

(c) 2020 S. Karger AG, Basel

\section{Introduction}

Congenital adrenal hyperplasia (CAH) is an autosomal recessive disease, which is due to 21-hydroxylase deficiency (21-OHD) caused by mutations in the cytochrome p450 family 21 subfamily A member 2 gene (CYP21A2) in more than $90 \%$ of patients [1]. Patients are

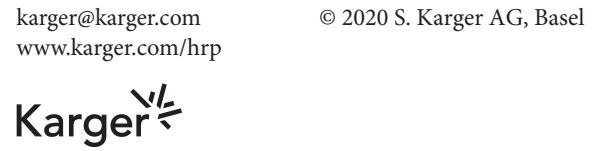

Marie L. Ljubicic

Department of Growth and Reproduction 
categorized into the classic and the non-classic form of $\mathrm{CAH}$. The severity correlates with the residual function of the 21-hydroxylase enzyme, so the mildest allele determines the phenotype of CAH with a good genotype/phenotype correlation, although divergence between genotype and phenotype has been reported [2-4]. Typically, classic $\mathrm{CAH}$ is associated with $<10 \%$ residual enzymatic activity [5], whereas patients with non-classic CAH carry genotypes that result in enzymatic activity corresponding to $30-50 \%$ [1]. Consequently, classic CAH is characterized by symptoms in the first years of life including salt wasting and atypical genitalia in girls due to severely decreased cortisol and aldosterone serum concentrations along with increased concentrations of androgens. Similarly, patients with non-classic $\mathrm{CAH}$ are characterized by later onset of symptoms such as premature adrenarche and androgenization due to less severe biochemistry as cortisol and aldosterone production is sufficient for everyday life, while adrenal androgens are elevated $[5,6]$.

Life-long replacement therapy with glucocorticoids, and mineralocorticoids for patients with salt-wasting, is therefore needed in most CAH patients to reduce the elevated concentrations of adrenal sex hormones. The therapeutic goal is to use the lowest possible doses of hydrocortisone and fludrocortisone to obtain a suppression of the adrenal androgens, while maintaining normal height and weight gain as well as pubertal development. To ensure sufficient treatment while hindering overtreatment, monitoring anthropometry including bone maturation as well as blood pressure frequently is the standard protocol. A recent study in fact recommended altering glucocorticoid doses according to pubertal development and anthropometric measurements [7].

Moreover, optimal management includes biochemical workups with measurements of 17-hydroxyprogesterone (17-OHP) and the androgen metabolites androstenedione, dehydroepiandrosterone-sulphate (DHEAS), and testosterone. Measuring serum concentrations of these hormones using liquid chromatography-tandem mass spectrometry (LC-MS/MS) is the gold standard $[8,9]$. Recently, we developed an improved method of LC-MS/MS that allows for simultaneous measurements of ten steroid metabolites in serum [10] and determined sex- and agespecific reference ranges based on 1,365 healthy children [11]. Marked variations due to sex and age exist for circulating levels of androgen metabolites as part of normal human physiology. Moreover, inter-center variations in analysis techniques and methods may exist, and altogether this complicates optimal treatment management. Theoretically, applying standard deviation (SD) scores of hormones rather than their absolute concentrations would eliminate differences based on sex, age, and center, thereby simplifying the interpretations of biochemical workups.

To the best of our knowledge, sex- and age-specific SD scores for serum 17-OHP and androgen metabolites (androstenedione, DHEAS, and testosterone) have not previously been applied in patients with CAH. Thus, in this single-center pilot study of 38 children and adolescents with $\mathrm{CAH}$ due to $21-\mathrm{OHD}$, the aim was to present these adrenal steroids expressed as SD scores to simplify research and in the future also simplify optimal management of treatment.

\section{Materials and Methods}

\section{Patients}

In this retrospective study, 43 children and adolescents under the age of 19 years with $\mathrm{CAH}$ due to $21-\mathrm{OHD}$ and followed at the Department of Growth and Reproduction, Copenhagen University Hospital, Copenhagen, Denmark, were identified as having 17-OHP and adrenal androgens (DHEAS, androstenedione and testosterone) determined by LC/MS-MS in the period from October 31, 2013, until May 1, 2018. Exclusion criteria were CAH due to other mutations than in CYP21A2 $(n=3)$ and missing data on the genetic cause of $\mathrm{CAH}(n=2)$. The final study population therefore included 38 patients ( 29 females, 9 males) ranging from 3.0 to 18.9 years of age at the last visit.

The 38 patients were routinely followed with an average of 3.5 visits (range 1.4-6.3) per year. Thus, an average of 11.5 longitudinal hormone measurements per patient in the study period (range 2-18) were included.

All patients were genotyped in a diagnostic setting. We categorized patients into 4 groups based on their CYP21A2 mutations according to Wedell et al. [2]. Thus, classic CAH included the following groups: complete enzyme impairment (residual enzyme activity of $0 \%$ ), almost complete enzyme impairment (the I2-splice mutation; a low but quantifiable residual activity), and severe enzyme impairment (the I173N mutation; residual activity of $2-10 \%$ ), while nonclassic CAH constituted one group (residual activity of 30-50\%) [1].

All biochemical and clinical data were collected as part of routine follow-up in the outpatient clinic, and the following data were collected from medical records corresponding to the follow-up period: 17-OHP and the androgens, clinical outcomes (blood pressure, bone age, and height), and treatment (types of medication and doses). All patients received treatment except for 1 female with non-classic $\mathrm{CAH}$ and a very mild phenotype with normal hormone concentrations.

\section{Hormone Analyses}

Serum concentrations of 17-OHP and androgens were determined by using the isotope-dilution TurboFlow-LC-MS/MS method as previously reported [10]. The limits of detection (LODs) were: $17-\mathrm{OHP}=0.10 \mathrm{nM}$, androstenedione $=0.04 \mathrm{nM}, \mathrm{DHEAS}=19 \mathrm{nM}$, and testosterone $=0.01 \mathrm{nM}$. Values below the LOD were replaced with the hormone-specific LOD divided by 2 . The inter-assay coef- 
Table 1. Distribution of sex and genotypes in 38 patients with congenital adrenal hyperplasia due to 21-hydroxylase deficiency

\begin{tabular}{|c|c|c|c|c|}
\hline \multirow[t]{2}{*}{ Group } & \multirow[t]{2}{*}{ Allele 1} & \multirow[t]{2}{*}{ Allele 2} & \multicolumn{2}{|l|}{$n$} \\
\hline & & & $\mathrm{F}$ & M \\
\hline \multicolumn{5}{|l|}{ Classic CAH $(n=19)$} \\
\hline \multirow{3}{*}{$\begin{array}{l}\text { Complete enzyme } \\
\text { impairment }(n=4)\end{array}$} & c. $955 \mathrm{C}>\mathrm{T}, \mathrm{p} .\left(\mathrm{G} \ln 319^{*}\right)$ & c. $955 \mathrm{C}>\mathrm{T}, \mathrm{p} .\left(\mathrm{G} \ln 319^{*}\right)$ & 1 & \\
\hline & Deletion (c.332_339del) & Deletion (c.332_339del) & 1 & 1 \\
\hline & Deletion (c.332_339del) & c.1069C>T, p.(Arg357Trp) & 1 & \\
\hline \multirow[t]{6}{*}{$\begin{array}{l}\text { Almost complete enzyme } \\
\text { impairment }(n=11)\end{array}$} & $\begin{array}{l}\text { c. } 293-13 \mathrm{C}>\mathrm{G} \text { (I2-Splice), } \\
\text { c. } 955 \mathrm{C}>\mathrm{T}, \mathrm{p} .\left(\mathrm{Gln} 319^{*}\right)\end{array}$ & Deletion (c.332_339del) & 1 & 1 \\
\hline & c.293-13C>G (I2-Splice) & Deletion (c.332_339del) & 4 & 1 \\
\hline & c.293-13C>G (I2-Splice) & c.1069C > T, p.(Arg357Trp) & & 1 \\
\hline & c.293-13C>G (I2-Splice) & c. $955 \mathrm{C}>\mathrm{T}, \mathrm{p} .\left(\mathrm{G} \ln 319^{*}\right)$ & 1 & \\
\hline & c.293-13C>G (I2-Splice) & c.293-13C>G (I2-Splice) & 1 & \\
\hline & I2 splice/del & NA & 1 & \\
\hline \multirow{3}{*}{$\begin{array}{l}\text { Severe enzyme impairment } \\
(n=4)\end{array}$} & c.518T>A, p.(Ile173Asn) & c.955C>T, p. $\left(\mathrm{G} \ln 319^{*}\right)$ & 1 & \\
\hline & c.518T >A, p.(Ile173Asn) & Deletion (c.332_339del) & 2 & \\
\hline & Ic.518T>A, p.(Ile173Asn) & c.293-13C>G (I2-Splice) & 1 & \\
\hline \multicolumn{5}{|l|}{ Non-classic CAH $(n=19)$} \\
\hline & c.92C>T, p.(Pro31Leu) & c. $955 \mathrm{C}>\mathrm{T}, \mathrm{p} .\left(\mathrm{G} \ln 319^{*}\right)$ & 1 & \\
\hline & c.92C>T, p.(Pro31Leu) & Deletion (c.332_339del) & 3 & 2 \\
\hline & c.92C>T, p.(Pro31Leu) & $\begin{array}{l}\text { c. }(710 \mathrm{~T}>\mathrm{A} ; 713 \mathrm{~T}>\mathrm{A} ; 719 \mathrm{~T}>\mathrm{A}), \mathrm{p} . \\
\text { (Ile237Asn; Val238Glu; Met240Lys) } \\
\text { Cluster E6) }\end{array}$ & 1 & \\
\hline & c.844G>T, p.(Val282Leu) & Deletion (c.332_339del) & 1 & \\
\hline & c.844G>T,p.(Val282Leu $)$ & c. $955 \mathrm{C}>\mathrm{T}, \mathrm{p} .\left(\mathrm{Gln} 319^{*}\right)$ & 1 & \\
\hline & c.1360C $>$ T, p.(Pro454Ser) & c. $955 \mathrm{C}>\mathrm{T}, \mathrm{p} .\left(\mathrm{G} \ln 319^{*}\right)$ & 1 & \\
\hline & c.1447C $>$ T, p.(Pro483Ser) & c. $955 \mathrm{C}>\mathrm{T}, \mathrm{p} .\left(\mathrm{G} \ln 319^{*}\right)$ & 1 & \\
\hline & c. $143 \mathrm{~A}>\mathrm{G}, \mathrm{p} .($ Tyr48Cys $)$ & c. $293-13 \mathrm{C}>\mathrm{G}$ (I2-Splice) & 1 & \\
\hline & c.844G>T, p.(Val282Leu) & c.293-13C>G (I2-Splice) & 1 & 1 \\
\hline & c.844G>T,p.(Val282Leu) & c.518T>A, p.(Ile173Asn) & & 1 \\
\hline & c.92C>T,p.(Pro31Leu) & c. $(710 \mathrm{~T}>\mathrm{A} ; 713 \mathrm{~T}>\mathrm{A} ; 719 \mathrm{~T}>\mathrm{A}$ & 1 & \\
\hline & & $\begin{array}{l}\text { c.844G > T), p.(Ile237Asn; Val238Glu; } \\
\text { Met240Lys; Val282Leu) }\end{array}$ & & \\
\hline & c.844G>T, p.(Val282Leu) & c.844G>T, p.(Val282Leu) & 3 & \\
\hline
\end{tabular}

$\mathrm{CAH}$, congenital adrenal hyperplasia; $\mathrm{F}$, female; $\mathrm{M}$, male.

ficients of variations were $1.9 \%$ for $17-\mathrm{OHP}, 1.4 \%$ for androstenedione, $2.4 \%$ for DHEAS, and $2.5 \%$ for testosterone [10]. All androgen analyses were performed at the Department of Growth and Reproduction, Copenhagen University Hospital, Copenhagen, Denmark. All steroid concentrations presented in the tables were calculated as medians of the SD scores. It was not possible to calculate DHEAS SD scores in patients between the age of 5 and 6.9 years due to lacking $\mathrm{L}, \mathrm{M}$, and $\mathrm{S}$ variables (see statistical analyses).

\section{Growth}

Height was measured to the nearest $0.5 \mathrm{~cm}$ using a wall-mounted Harpenden stadiometer, while weights were measured to the nearest $0.1 \mathrm{~kg}$ using calibrated electronic scales. Age-adjusted SD scores for height, weight, and BMI were calculated using national references [12]. Bone age was determined by automated bone age reading (BoneXpert) of an X-ray image of the hand [13]. Bone ageadjusted height was determined by using bone age rather than chronological age for the calculation of SD scores, also using the national references [12]. Bone maturation was expressed as bone age minus chronological age, both in years. A positive value indicated that bone age was greater than chronological age (advanced bone age), and a negative value indicated that bone age was less than chronological age (bone age delay). Final height was deemed "final" in patients where 3 or more consecutive measurements over a minimum of 1.5 years were stable $( \pm 1 \mathrm{~cm})$. Final height SD scores were calculated with a fictional age of 20 years to avoid overestimating the SD scores due to early growth spurts. Target height was calculated as the mean of maternal and paternal height SD scores and back-transformed to sex-specific heights (in centimeters) without correction for secular trend [13]. Height and final height 
Table 2. Descriptive data in 38 patients with congenital adrenal hyperplasia $(\mathrm{CAH})$ due to 21-hydroxylase deficiency

\begin{tabular}{|c|c|c|c|c|c|c|}
\hline & \multicolumn{2}{|c|}{ All CAH patients $(n=38)$} & \multicolumn{2}{|c|}{ Classic CAH $(n=19)$} & \multicolumn{2}{|c|}{ Non-classic CAH $(n=19)$} \\
\hline & $n$ & median (range) & $n$ & median (range) & $n$ & median (range) \\
\hline \multicolumn{7}{|l|}{ Anthropometry } \\
\hline Height, age-adjusted SDS & 37 & $-0.04(-2.69$ to 2.60$)$ & 19 & $-0.34(-2.69$ to 2.60$)$ & 18 & 0.27 (-2.02 to 2.48$)$ \\
\hline Height, bone age-adjusted SDS & 35 & $-0.49(-2.41 \text { to } 1.77)^{*}$ & 17 & $-0.62(-2.41 \text { to } 1.77)^{*}$ & 18 & $-0.42(-2.38$ to 1.71$)$ \\
\hline Bone maturation, years & 35 & $0.87(-1.51 \text { to } 4.49)^{* *}$ & 17 & $0.41(-1.28$ to 3.94$)$ & 18 & $1.25(-1.51 \text { to } 4.49)^{* *}$ \\
\hline Final height SDS & 15 & $-0.34(-2.02$ to 0.78$)$ & 7 & $0.34(-2.02$ to 0.06$)$ & 8 & $-0.38(-1.82$ to .078$)$ \\
\hline Final height SDS minus TH SDS & 15 & $-0.28(-2.36$ to 1.33$)$ & 7 & $-0.68(-2.36$ to 1.04$)$ & 8 & $-0.17(-2.06$ to 1.33$)$ \\
\hline Weight SDS & 38 & $0.81(-2.82 \text { to } 2.73)^{*}$ & 19 & $0.41(-2.82$ to 2.73$)$ & 19 & $1.15(-1.65 \text { to } 2.46)^{*}$ \\
\hline BMI SDS & 38 & $0.79(-1.30 \text { to }-0.24)^{* *}$ & 19 & $0.80(-1.30 \text { to } 3.29)^{*}$ & 19 & $0.79(-1.08 \text { to } 2.67)^{*}$ \\
\hline Hydrocortisone, $\mathrm{mg} / \mathrm{m}^{2}$ & 37 & $12.6(0$ to 20.6$)$ & 19 & $13.4(8.9$ to 20.6$)$ & 18 & $11.5(0.0$ to 20.4$)$ \\
\hline Fludrocortisone, $\mu \mathrm{g}$ & 17 & $70(20$ to 150$)$ & 14 & $65(20$ to 125$)$ & 4 & $65(30$ to 150$)$ \\
\hline
\end{tabular}

Data are shown for the total group and divided into classic and non-classic forms. Anthropometric and treatment medians and ranges are based on one median per variable per patient. AAH, age-adjusted height; $\mathrm{BAH}$, bone age-adjusted height; bone maturation, calculated as bone age minus chronological age; DBP, diastolic blood pressure; $\mathrm{mg} / \mathrm{m}^{2}$, milligram per square meter body surface area; range, minimum and maximum values; SBP, systolic blood pressure; SDS, standard deviation score; TH, target height. Significance level of $p$ values expressed by ${ }^{*}<0.05$, ${ }^{* *}<0.005$, and ${ }^{* * *}<0.001$.

SD scores (chronological age-adjusted) and height SD scores (bone age-adjusted) were corrected for target height via simple subtraction: height SD score - target height SD score and final height SD score - target height SD score. Negative values indicated growth below genetic potential while positive values indicated growth above genetic potential.

\section{Blood Pressure}

Blood pressure was measured in a supine position. Systolic and diastolic blood pressure (SBP and DBP) measurements were expressed as SD scores using the references reported by Neuhauser et al. [14]. SBP and DBP SD scores were expressed as medians of all measurements for each patient.

\section{Hormone Replacement Therapy}

Doses of hydrocortisone were reported in milligrams per square meter of body surface area per day $\left(\mathrm{mg} / \mathrm{m}^{2} /\right.$ day $)$ and doses of fludrocortisone were reported in micrograms per day ( $\mu \mathrm{g} /$ day). Data were compared to the recommended maintenance doses of hydrocortisone (10-15 mg/m²/day) and fludrocortisone (100-200 $\mu \mathrm{g} /$ day) [10]. For each patient, data on multiple doses were converted to a single median.

\section{Statistical Analyses}

The distributions of 17-OHP, androstenedione, DHEAS, and testosterone serum concentrations in healthy children and adolescents according to age and sex have previously been reported using a generalized additive model for location, shape and scale (GAMLSS) [11]. In this study, individual SD scores for serum lev- els were calculated using GAMLSS statistics that transforms data to follow a parametric distribution by the equation: $\mathrm{SD}$ score = $\left((\mathrm{X} / \mathrm{M})^{\mathrm{L}}-1\right) /(\mathrm{L} \times \mathrm{S})$, where $\mathrm{X}$ is the measurement, $\mathrm{L}$ adjusts for sex- and age-dependent skewness $(L \neq 0), M$ corresponds to the sex- and age-dependent median, and $\mathrm{S}$ approximates the sex- and age-dependent coefficient of variation.

SD scores for SBP and DBP were calculated using the reference data from Neuhauser et al. [14]. The provided standardized blood pressure measurements in children and adolescents from the age of 3 to 17 years, which simultaneously account for age and height, were applied to calculate SD scores using GAMLSS statistics.

Each patient had median SD scores calculated for 17-OHP and androgen measurements and clinical outcomes to avoid single patients skewing the data set with more visits. Median SD scores of androgen measurements and clinical outcomes were compared to zero (reference material) using the one-sample Wilcoxon signed rank test. SD scores ranging from -2 to 2 were considered normal. $p$ values $<0.05$ were considered statistically significant. All statistical analyses were performed using IBM Statistics SPSS, version 22.

\section{Results}

\section{Clinical Data}

All 38 patients were grouped according to previous mutation-based predictions of residual enzymatic activity into classical CAH with complete enzyme impairment 


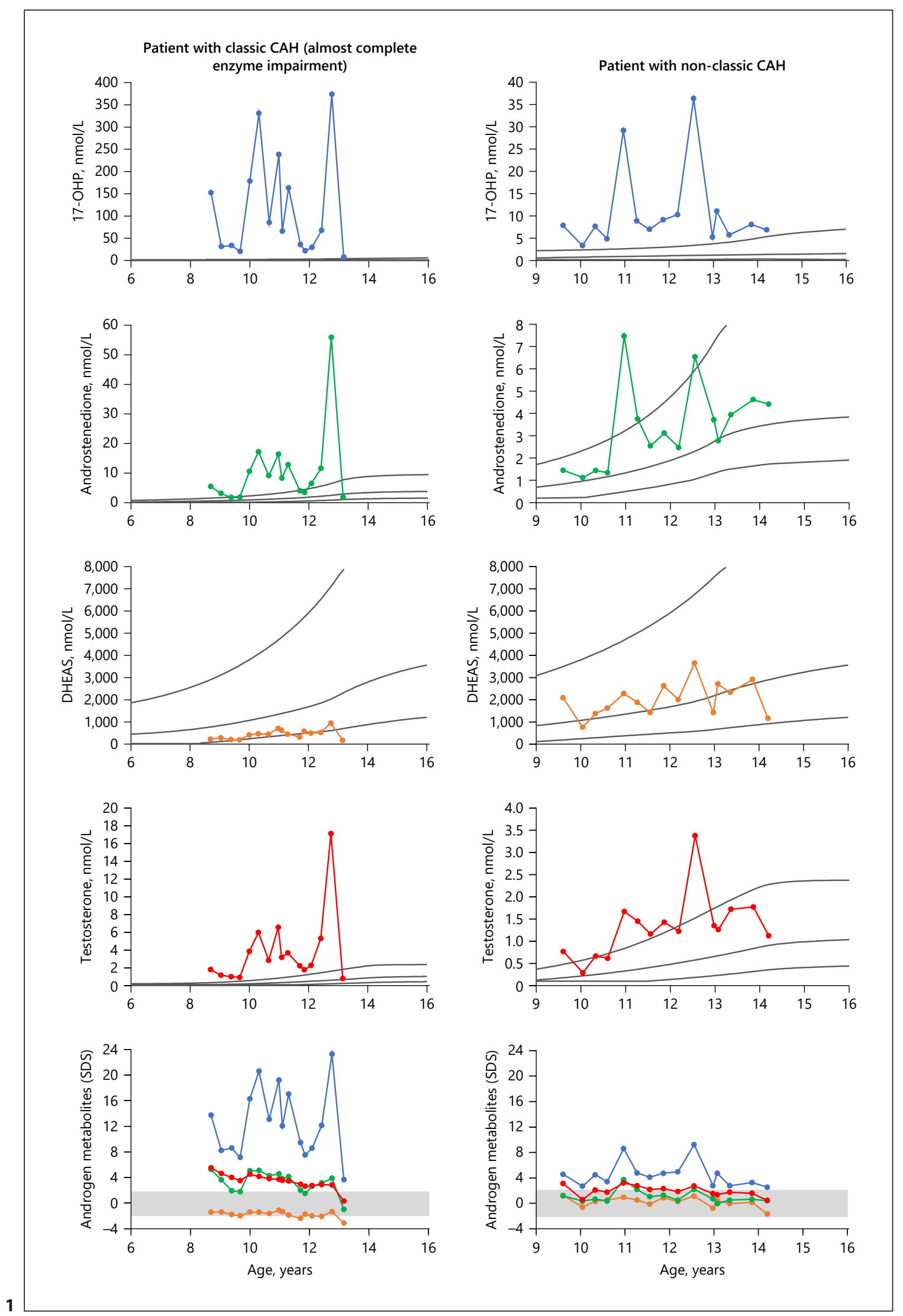

(For legend see next page.) 
Table 3. Concentrations of 17-hydroxyprogestone and androgen metabolites expressed as standard deviation scores in 38 patients with congenital adrenal hyperplasia due to 21-hydroxylase deficiency

\begin{tabular}{|c|c|c|c|c|c|c|}
\hline & \multicolumn{2}{|c|}{ All CAH patients $(n=38)$} & \multicolumn{2}{|c|}{ Classic CAH $(n=19)$} & \multicolumn{2}{|c|}{ Non-classic CAH $(n=19)$} \\
\hline Androstenedione & 37 & $0.61(-4.45$ to 3.71$)$ & 18 & $0.00(-1.83$ to 3.71$)$ & 19 & $0.90(-4.45$ to 2.34$)$ \\
\hline DHEAS & 36 & $-1.78(-3.87 \text { to } 1.62)^{* * *}$ & 17 & $-2.43(-3.87 \text { to }-1.65)^{* * *}$ & 19 & $0.11(-3.83$ to 1.62$)$ \\
\hline Testosterone & 37 & $1.12(-4.14 \text { to } 5.65)^{* * *}$ & 18 & $0.68(-1.92$ to 5.65$)$ & 19 & $1.85(-4.14 \text { to } 3.76)^{* *}$ \\
\hline
\end{tabular}

Data are shown for the total group and divided into classic and non-classic forms. Metabolite medians and ranges are based on one median per metabolite per patient. CAH, congenital adrenal hyperplasia; DHEAS, dehydroepiandrosterone sulphate; 17-OHP, 17-hydroxyprogestone. Significance level of $p$ values expressed by $*<0.05, * *<0.005$, and ${ }^{* * *}<0.001$.

$(n=4)$, almost complete enzyme impairment $(n=11)$, and severe enzyme impairment $(n=4)$, and into nonclassic CAH $(n=19)$ (Table 1$)$.

Clinical outcomes are summarized in Table 2. Heights in CAH patients (overall and in both groups) did not differ significantly from the reference material either when age-adjusted or in terms of final height. This was also true for height and final height corrected for target height (all $p$ values were $>0.05$ ). Compared to the reference material, height adjusted for bone age was significantly lower in $\mathrm{CAH}$ overall $(p=0.009)$ as well as in classic CAH $(p=$ 0.04 ), while bone age-adjusted height corrected for target height did not significantly differ from the reference material either overall or for each $\mathrm{CAH}$ group (median SD score: 0.00 , range -2.73 to $2.25, p>0.05$ for all CAH patients). However, bone age was significantly advanced compared to chronological age (median difference: 0.87 years, range -1.51 to $4.49, p<0.005)$. Weight SD scores were significantly higher in the overall CAH group ( $p=$ $0.02)$ and in non-classic patients $(p=0.03)$ than in the reference material. Additionally, BMI SD scores were significantly higher in the overall CAH group $(p=0.001)$ and in both classic $(p=0.03)$ and non-classic patients $(p=0.01)$ than in the reference material.

SBP SD scores (median SD score: 0.44 , range -1.14 to 1.56) and DBP SD scores (median SD score: 0.91,

Fig. 1. Serum concentrations of 17-hydroxyprogesterone (17-OHP), androstenedione, dehydroepiandrosterone sulphate (DHEAS), and testosterone shown as absolute concentrations and as SD scores in 2 patients with classic congenital adrenal hyperplasia (CAH) and complete enzyme impairment and non-classic $\mathrm{CAH}$ due to 21-hydroxylase deficiency. Grey lines: mean \pm 2 SD scores. Hatched areas: \pm 2 SD scores. SDS, standard deviation scores.

Steroid Hormone SD Scores in Children with $\mathrm{CAH}$ range -1.21 to 2.13 ) were significantly elevated compared to the reference range $(p<0.01$ and $p<0.001$, respectively).

The median hydrocortisone dose was $12.6 \mathrm{mg} / \mathrm{m}^{2} /$ day $\left(0-20.6 \mathrm{mg} / \mathrm{m}^{2} /\right.$ day $)$, and $5 \%(n=2)$ received doses exceeding $20 \mathrm{mg} / \mathrm{m}^{2} /$ day. Furthermore, $45 \%(n=17)$ also received fludrocortisone, with a median dose of $70 \mu \mathrm{g} /$ day (20-150 $\mu \mathrm{g} /$ day).

\section{7-OHP, Androgen, and Renin Concentrations}

Absolute hormone concentrations and relative values expressed as SD scores are shown in 2 representative patients (classic $\mathrm{CAH}$ with complete enzyme impairment and non-classic $\mathrm{CAH}$, respectively) in Figure 1.

In all patients, 17-OHP serum concentrations were significantly increased when compared to the reference both overall and when grouped into classic and non-classic CAH $(p<0.001$; Table 3$)$. In total, $86 \%(n=32)$ of $\mathrm{CAH}$ patients had 17-OHP measurements above $+2 \mathrm{SD}$, while $11 \%(n=4)$ had $17-O H P$ within the normal range, and $3 \%(n=1)$ had 17-OHP concentrations below -2 SD (Fig. 2a).

Androstenedione concentrations did not differ from the reference in either classic or non-classic CAH $(p \geq$ $0.11)$. In total, $11 \%(n=4)$ had androstenedione measurements above +2 SD, $86 \%$ of patients $(n=32)$ had them within the normal range, and 3\% $(n=1)$ had measurements below $-2 \mathrm{SD}$ (Fig. 2a).

DHEAS concentrations were significantly decreased in the children with $\mathrm{CAH}$ compared to the reference; however, they were only statistically significantly lowered in children with classic CAH ( $p<0.001$; Table 3$)$. In total, $53 \%(n=19)$ of patients had DHEAS measurements within the normal range, and $47 \%(n=17)$ had DHEAS mea- 


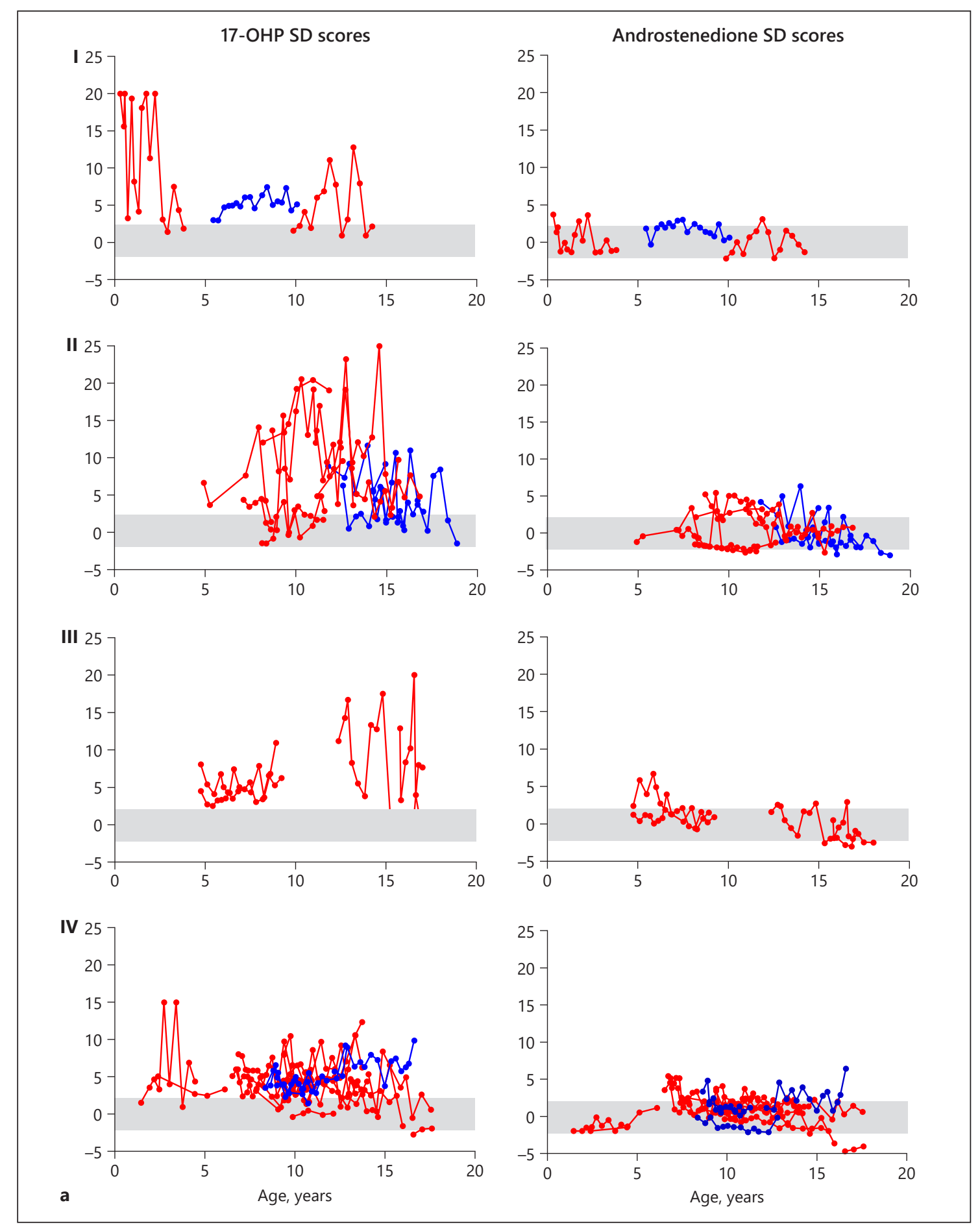

Fig. 2. a Longitudinal serum concentrations of 17-hydroxyprogesterone (17-OHP; left panel) and androstenedione (right panel) expressed as SD scores in patients with congenital adrenal hyperplasia $(\mathrm{CAH})$. Patients are grouped according to genotype into classic CAH due to (I) complete enzyme impairment, (II) almost complete enzyme impairment, and (III) severe enzyme impairment, and into (IV) non-classic CAH. Boys are marked in blue and girls in red. Hatched areas: \pm 2 SD scores. $\mathbf{b}$ Longitudinal serum concen- trations of dehydroepiandrosterone sulphate (DHEAS; left panel) and testosterone (right panel) expressed as SD scores in patients with congenital adrenal hyperplasia. Patients are grouped according to genotype into classic CAH due to (I) complete enzyme impairment, (II) almost complete enzyme impairment, and (III) severe enzyme impairment, and into (IV) non-classic CAH. Boys are marked in blue and girls in red. Hatched areas: \pm 2 SD scores.

(Figure continued on next page.) 


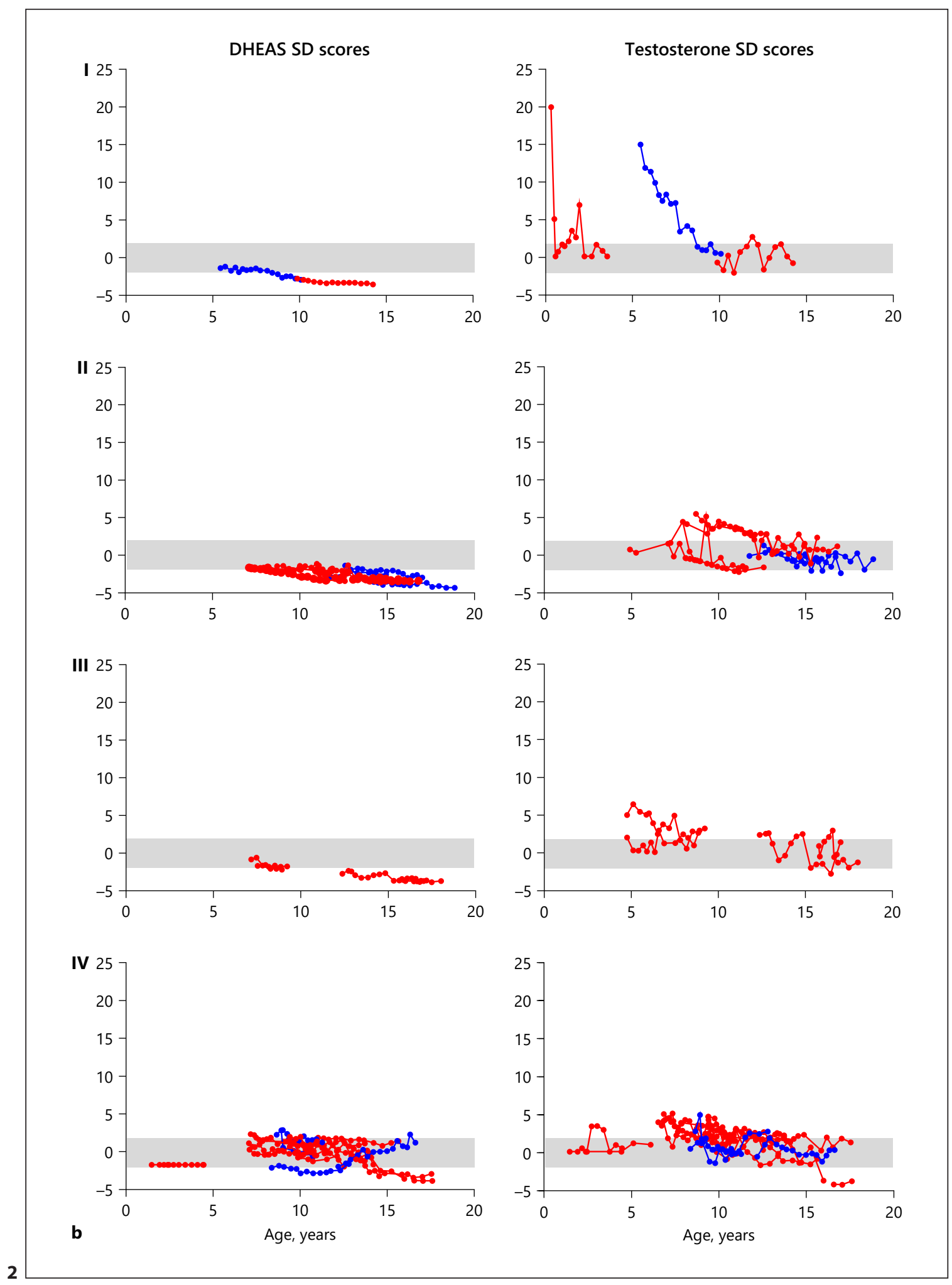



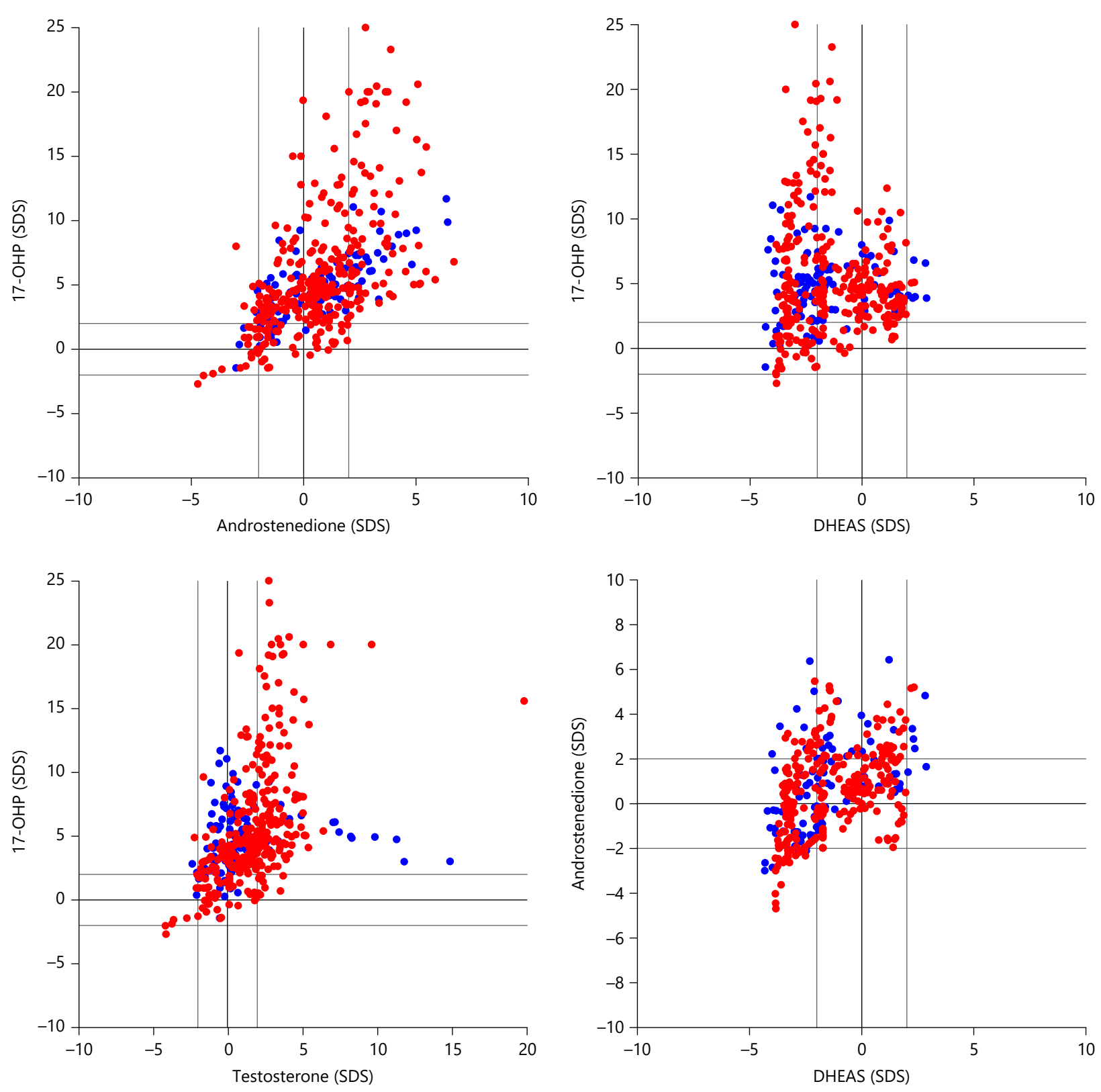

Fig. 3. Serum concentrations of 17-hydroxyprogesterone (17$\mathrm{OHP}$ ) SD scores as a function of androstenedione, dehydroepiandrosterone sulphate (DHEAS), and testosterone, respectively, and

surements below $-2 \mathrm{SD}$. None of the patients had DHEAS concentrations above +2 SD (Fig. 2b).

Testosterone concentrations were significantly increased in CAH patients overall $(p<0.001)$; however, they were only significantly increased in patients with androstenedione SD scores as a function of DHEAS SD scores in patients with congenital adrenal hyperplasia. Grey lines: \pm 2 SD scores. SDS, standard deviation scores. 
The associations between the SD scores of the 4 hormones are shown as bivariate plots in Figure 3. Visually, most androstenedione concentrations were within the normal range ( $-2 \mathrm{SD}$ to $+2 \mathrm{SD})$, while the majority of the corresponding 17-OHP concentrations were above +2 SD. Similar patterns were seen with $17-\mathrm{OHP}$ versus DHEAS and testosterone. All $4 \mathrm{CAH}$ subgroups had androstenedione SD scores lower than the corresponding 17-OHP SD scores (data not shown).

Median renin concentrations in absolute values did not differ between patients with classic (median: 53.8 $\mathrm{IU} / \mathrm{L}$, range 3.4-261.5 IU/L) and non-classic CAH (median: $32.5 \mathrm{IU} / \mathrm{L}$, range $2.2-118.5 \mathrm{IU} / \mathrm{L}, p=0.07$ ).

\section{Discussion}

This pilot study, from a single center, of 38 children and adolescents with $\mathrm{CAH}$ due to 21-OHD presents adrenal steroid metabolite concentrations expressed as sexand age-dependent SD scores using the state-of-the-art measurement technique LC-MS/MS.

To our knowledge, the expression of androgen metabolites measured in serum as sex- and age-related SD scores in a pediatric CAH population has not been evaluated before. Moreover, in the recent Endocrine Society Guidelines for CAH management, it was noted that optimal ranges for biochemical markers are impossible due to variances within sex, ages, laboratory assays, and reference ranges [9]. By applying SD scores in this study, we eliminated differences based on sex and age. More specifically, this tool allows for comparison across these factors independent of assay and center. For example, a concentration of androstenedione of $3 \mathrm{nmol} / \mathrm{L}$ in a girl does not reflect identical levels when evaluated against normal ranges in a 10-year-old and in a 12-year-old. In this case, the SD scores are 2.7 and 1.0, respectively, thus illustrating that the same absolute concentration is above the normal range in a 10 -year-old but within the normal range in a 12-year-old. Adding different sexes and assays, comparison of absolute concentrations is only more difficult. Applying SD scores therefore allows for clinical characteristics and treatment of patients to be compared independent of where and how the individual patient's biochemistry is monitored. It is, however, important to note that currently optimal ranges for 17-OHP and adrenal androgens have not been established for children and adolescents and the direct application and use of SD scores in the clinical setting is not yet possible. However, application of SD scores in com-

Steroid Hormone SD Scores in Children with $\mathrm{CAH}$ parisons of research projects across centers is possible; in fact, we recommend that SD scores be used in these instances. This would also allow centers to compare their data on CAH patients with those published in the literature.

The converted absolute concentrations of 17-OHP, androstenedione, DHEAS, and testosterone to sex- and age-related SD scores in this study revealed that overall, 17-OHP concentrations were significantly elevated, while androstenedione scores were within the normal range in the majority of children. Thus, 17-OHP levels were overall higher than androstenedione levels. This is somewhat in accordance with the guidelines that noted that 17-OHP and androstenedione are often high-normal to mildly elevated in sufficiently treated CAH patients [9]. Furthermore, testosterone was also elevated in many patients in this study. However, to our knowledge, testosterone concentrations are best applied to monitor treatment only in adults with $\mathrm{CAH}$ [9], and further discussion of testosterone is therefore beyond the scope of this study. Interestingly, DHEAS was significantly decreased. This has been reported previously [15], and DHEAS has thus been suggested as a marker of non-compliance as subnormal/low concentrations reflect acceptable treatment [6]. Moreover, the urinary metabolome in pediatric $\mathrm{CAH}$ patients has previously been evaluated using SD scores denoting the same advantages [16]. The same study furthermore found elevated concentrations of 17-OHP and normal concentrations of DHEA in urine, while also noting that androgen excess in treated patients with $\mathrm{CAH}$ was primarily due to adrenal 11-oxygenated C19 steroids. These 11 -oxygenated C19 steroids can be measured in serum as well [17], but measurement of these steroids was not possible in our laboratory, so we were unable to confirm these results in our study population.

When interpreting the biochemical SD scores in $\mathrm{CAH}$ patients, it is important to keep the timing of sampling in mind. In fact, variations due to medical treatment and diurnal fluctuations of steroid hormones have previously been reported in patients with CAH [18] and consequently consistent sampling in the morning is recommended [9]. Additionally, for patients with non-classic CAH it is also important to bear in mind that in general they have milder phenotypes and therefore may have hormone SD scores closer to normal. Fifty percent of our study population was made up of patients with non-classic $\mathrm{CAH}$; reported overall SD scores may thus be overestimations for the non-classic $\mathrm{CAH}$ patients and underestimations for the classic $\mathrm{CAH}$ patients. However, CAH patients, classic and non-classic, make up a heterogeneous group, and hormone SD scores 
may thus be subject to individual variables such as time of diagnosis, genotype, and compliance to treatment.

Management and monitoring of CAH patients is generally accepted to include virilization and premature onset of puberty, anthropometry, and blood pressure [7, 9]. To describe our patient population, we therefore evaluated particularly anthropometry and blood pressure at a group level in this study. Overall, when correcting for both bone age and target height, the patients in the study had normal heights. This was confirmed by normal final heights when corrected for target heights. Although bone age was significantly advanced, particularly in the non-classic group, we concluded that overall, the patients in this study grew almost according to genetic potential (median SD scores were only slightly below 0 ). This is in line with a previous report that patients with $\mathrm{CAH}$ who undergo strict medicine and follow-up regimens achieve final height near target height [19]. Final height below target height although within \pm 2 SD has, nonetheless, been most frequently reported [20]. Thus, one of the aims of treatment is to normalize the growth pattern and achieve a final height within genetic potential [9]. Moreover, many patients in our cohort had high BMIs ( $32 \%$ above +1 SD and $8 \%$ above +2 $\mathrm{SD})$, namely in the non-classic group. The WHO defines BMI above $+1 \mathrm{SD}$ as overweight and above $+2 \mathrm{SD}$ as obesity [21]. Overweight and obesity have also previously been reported in the literature [9], and a larger study observed obesity in $17 \%$ of children and adolescents with CAH [22]. Lastly, $48 \%$ of patients had systolic hypertension and $32 \%$ had diastolic hypertension on one or more occasions, which is in line with previous reports on children and adolescents with $\mathrm{CAH}[23,24]$.

Anthropometric data and blood pressure measurements are important markers of treatment and disease control in all patients with CAH. The patients in this study were treated with an average hydrocortisone dose of 12.6 $\mathrm{mg} / \mathrm{m}^{2} /$ day, and 17 patients also received fludrocortisone acetate; the doses of hydrocortisone were in accordance with the guidelines [9]. It is noteworthy that 4 patients with non-classic $\mathrm{CAH}$ received fludrocortisone which, albeit not included in the guidelines, has been described previously as a means to minimize glucocorticoid doses [6]. In terms of growth, the patients in our study seemed adequately treated, although factors such as the number of blood pressure measurements within the hypertensive range as well as the number of overweight patients can lead to questions regarding this. However, the elevated levels of 17-OHP in our patients further indicate sufficient treatment as suggested by the guidelines [9]. Overall, it is important to evaluate each patient individually; there are patients in whom abnormal anthropometry or blood pressure, as well as skewed biochemical markers, should spark questions regarding medicine compliance. Moreover, in our study, it was noteworthy that all patients with nonclassic $\mathrm{CAH}$, except for one, received treatment during the follow-up period. In general, it is recommended to treat non-classic CAH children and adolescents if they have inappropriately early signs of puberty or virilization, altered anthropometry for sex and age, abnormal blood pressure, or altered adrenal biochemistry [9]. Patients with nonclassic CAH in this study had remarkably normal growth; it can be speculated whether this is due to treatment or due to mild phenotypes. However, a randomized controlled trial would need to take place in order to differentiate.

Importantly, the focus of this study was on SD scores of adrenal biochemical markers and how they can be applied and evaluated, independent of treatment status and developmental stage in any patient. We believe that the application of SD scores by expressing optimal ranges of 17-OHP and adrenal androgens in SD scores would simplify monitoring of hydrocortisone treatment. Moreover, it allows for immediate comparisons across sex, ages, and centers (laboratories and assays). Thus, guidelines on CAH would be improved by including SD scores for 17 OHP and all androgen metabolites as they can be directly used in any clinic with a local reference range. Moreover, the inclusion would also allow for research to take place across centers and assays into hitherto unused metabolites as new markers of optimal treatment. It is, however, important to note that we do not propose that monitoring of children and adolescents with CAH be done by biochemistry alone; however, we do believe that if optimal ranges of the hormones were reported in SD scores, the biochemical part of the monitoring would be easier and more consistent.

The strengths of this study were that (1) the application of age- and sex-related SD scores enables data comparable across sex, age, assay, and center, (2) 17-OHP and all androgen metabolites were measured by the LC-MS/ MS method, which is considered the state-of-the-art method, (3) all patients were followed in a single, tertiary center during a limited time span, which reduces biases due to different protocols, and (4) all patients suffered from 21-OHD, making their outcomes comparable. The limitations of the study included that (1) the use of age-, sex- and assay-specific SD scores requires that each center calculates a reference interval to implement the SD scores in their clinic, (2) the random timing of blood samples in the outpatient clinic may be affected by diurnal variations and medication which are not accounted for in SD scores, 
(3) clinical as well as biochemical parameters were expressed as medians for each patient to avoid single patients skewing the data set due to more visits than others (but this also simplifies the data), (4) it was a small study population $(n=38)$, although this did not affect the use of SD scores, (5) the retrospective design of the study led to some missing clinical data in some patients, and (6) plasma renin activity was not routinely collected and pediatric reference ranges do not exist in our tertiary center and were thus not included in this study.

In conclusion, based on this pilot study, we propose that 17-OHP and androgen metabolite concentrations should be expressed as sex- and age-related SD scores, as it allows for comparison of androgen metabolite concentrations independent of sex, age, assay, and center. The integration of SD scores would simplify optimal patient management in $\mathrm{CAH}$ children and adolescents if also included in optimal ranges in future guidelines.

\section{Acknowledgment}

The authors would like to thank the patients who participated in this study and the staff at the outpatient clinic and in the laboratories involved in this study.

\section{Statement of Ethics}

This study was approved by The Danish Data Protection Agency (RH-2015-235, I-Suite No. 04204). The use of patient data was approved by the Danish Health and Medical Authorities (3-30131376/1).

\section{Disclosure Statement}

There is no conflict of interest that could be perceived as prejudicing the impartiality of the research reported.

\section{Funding Sources}

Marie L. Ljubicic was supported by The Absalon Foundation.

\section{Author Contributions}

C.S.C, M.L.L., T.H.J., and A.J. partook in the conception of the project, the acquisition of data, data analysis, and writing and approving the manuscript. J.H.P. partook in the conception of the project, data analysis, and writing and approving the manuscript. M.D. partook in data acquisition, data analysis, and writing and approving the manuscript. K.M.M., A.-M.A., and H.F. partook in data analysis and writing and approving the manuscript.

\section{References}

1 Wedell A. Molecular genetics of 21-hydroxylase deficiency. Endocr Dev. 2011;20:80-7.

2 Wedell A, Thilén A, Ritzén EM, Stengler B, Luthman H. Mutational spectrum of the steroid 21-hydroxylase gene in Sweden: implications for genetic diagnosis and association with disease manifestation. J Clin Endocrinol Metab. 1994 May;78(5):1145-52.

3 Krone N, Rose IT, Willis DS, Hodson J, Wild SH, Doherty EJ, et al.; United Kingdom Congenital adrenal Hyperplasia Adult Study Executive (CaHASE). Genotype-phenotype correlation in 153 adult patients with congenital adrenal hyperplasia due to 21-hydroxylase deficiency: analysis of the United Kingdom Congenital adrenal Hyperplasia Adult Study Executive (CaHASE) cohort. J Clin Endocrinol Metab. 2013 Feb;98(2):E346-54

4 New MI, Abraham M, Gonzalez B, Dumic M, Razzaghy-Azar M, Chitayat D, et al. Genotype-phenotype correlation in 1,507 families with congenital adrenal hyperplasia owing to 21-hydroxylase deficiency. Proc Natl Acad Sci USA. 2013 Feb;110(7):2611-6.

5 Speiser PW, White PC. Congenital adrenal hyperplasia. N Engl J Med. 2003 Aug;349(8): $776-88$.
6 Nordenstrom A, Falhammar H. MANAGEMENT OF ENDOCRINE DISEASE: diagnosis and management of the patient with nonclassic $\mathrm{CAH}$ due to 21-hydroxylase deficiency. Eur J Endocrinol. 2018 Dec;180(3):R127-45.

7 Pijnenburg-Kleizen KJ, Thomas CM, Otten BJ, Roeleveld N, Claahsen-van der Grinten HL. Long-term follow-up of children with classic congenital adrenal hyperplasia: suggestions for age dependent treatment in childhood and puberty. J Pediatr Endocrinol Metab. 2019 Oct;32(10):1055-63.

8 Bae YJ, Zeidler R, Baber R, Vogel M, Wirkner $\mathrm{K}$, Loeffler $\mathrm{M}$, et al. Reference intervals of nine steroid hormones over the life-span analyzed by LC-MS/MS: effect of age, gender, puberty, and oral contraceptives. J Steroid Biochem Mol Biol. 2019 Oct;193(Jun):105409.

9 Speiser PW, Arlt W, Auchus RJ, Baskin LS, Conway GS, Merke DP, et al. Congenital adrenal hyperplasia due to steroid 21-hydroxylase deficiency: an Endocrine Society clinical practice guideline. J Clin Endocrinol Metab. 2018 Nov; 103(11):4043-88.

10 Søeborg T, Frederiksen H, Johannsen TH, Andersson AM, Juul A. Isotope-dilution TurboFlow-LC-MS/MS method for simultaneous quantification of ten steroid metabolites in serum. Clin Chim Acta. 2017 May;468: $180-6$.
11 Søeborg T, Frederiksen H, Mouritsen A, Johannsen TH, Main KM, Jørgensen N, et al. Sex, age, pubertal development and use of oral contraceptives in relation to serum concentrations of DHEA, DHEAS, 17a-hydroxyprogesterone, $\Delta 4$-androstenedione, testosterone and their ratios in children, adolescents and young adults. Clin Chim Acta. 2014 Nov;437:6-13.

12 Tinggaard J, Aksglaede L, Sørensen K, Mouritsen A, Wohlfahrt-Veje C, Hagen CP, et al. The 2014 Danish references from birth to 20 years for height, weight and body mass index. Acta Paediatr. 2014 Feb;103(2):214-24.

13 Thodberg HH, Kreiborg S, Juul A, Pedersen KD. The BoneXpert method for automated determination of skeletal maturity. IEEE Trans Med Imaging. 2009 Jan;28(1):52-66.

14 Neuhauser HK, Thamm M, Ellert U, Hense HW, Rosario AS. Blood pressure percentiles by age and height from nonoverweight children and adolescents in Germany. Pediatrics. 2011 Apr;127(4):e978-88.

15 Falhammar H, Filipsson H, Holmdahl G, Janson PO, Nordenskjöld A, Hagenfeldt K, et al. Metabolic profile and body composition in adult women with congenital adrenal hyperplasia due to 21-hydroxylase deficiency. J Clin Endocrinol Metab. 2007 Jan;92(1):110-6. 
16 Kamrath C, Wettstaedt L, Boettcher C, Hartmann MF, Wudy SA. Androgen excess is due to elevated 11-oxygenated androgens in treated children with congenital adrenal hyperplasia. J Steroid Biochem Mol Biol. 2018;178: 221-8.

17 Turcu AF, Nanba AT, Chomic R, Upadhyay SK, Giordano TJ, Shields JJ, et al. Adrenal-derived 11-oxygenated 19-carbon steroids are the dominant androgens in classic 21-hydroxylase deficiency. Eur J Endocrinol. 2016 May;174(5):601-9.

18 Debono M, Mallappa A, Gounden V, Nella AA, Harrison RF, Crutchfield CA, et al. Hormonal circadian rhythms in patients with congenital adrenal hyperplasia: identifying optimal monitoring times and novel disease biomarkers. Eur J Endocrinol. 2015 Dec; 173(6):727-37.

19 Hoepffner W, Kaufhold A, Willgerodt H, Keller E. Patients with classic congenital adrenal hyperplasia due to 21-hydroxylase deficiency can achieve their target height: the Leipzig experience. Horm Res. 2008;70(1): 42-50.

20 Nebesio TD, Eugster EA. Growth and Reproductive Outcomes in Congenital Adrenal Hyperplasia. Int J Pediatr Endocrinol. 2010; 2010:298937.

21 de Onis M, Onyango AW, Borghi E, Siyam A, Nishida C, Siekmann J. Development of a WHO growth reference for school-aged children and adolescents. Bull World Health Organ. 2007 Sep;85(9):660-7.
22 Völkl TM, Simm D, Beier C, Dörr HG. Obesity among children and adolescents with classic congenital adrenal hyperplasia due to 21-hydroxylase deficiency. Pediatrics. 2006 Jan;117(1):e98-105.

23 Roche EF, Charmandari E, Dattani MT, Hindmarsh PC. Blood pressure in children and adolescents with congenital adrenal hyperplasia (21-hydroxylase deficiency): a preliminary report. Clin Endocrinol (Oxf). 2003 May;58(5):589-96.

24 Mooij CF, van Herwaarden AE, Sweep FC, Roeleveld N, de Korte CL, Kapusta L, et al. Cardiovascular and metabolic risk in pediatric patients with congenital adrenal hyperplasia due to 21 hydroxylase deficiency. J Pediatr Endocrinol Metab. 2017 Aug;30(9):957-66. 